\title{
Cepheid mass-luminosity relations from the Magellanic Clouds
}

\author{
J. P. Beaulieu ${ }^{1}$, J. R. Buchler ${ }^{1,2}$, and Z. Kolláth ${ }^{1,3}$ \\ 1 Institut d'Astrophysique de Paris, 98bis boulevard Arago, 75014 Paris, France \\ 2 Department of Physics, University of Florida, Gainesville, Fl 32611, USA \\ 3 Konkoly Observatory, POB 67, Budapest, 1525 Hungary
}

Received 24 October 2000 / Accepted 5 March 2001

\begin{abstract}
The OGLE data base is used in conjunction with Kurucz atmosphere models to generate sets of period, effective temperature and luminosity for fundamental and overtone Magellanic Cloud Cepheids. The Florida pulsation code (with linear turbulent convection) is then used to compute masses for these stars, assuming an average composition of $(X=0.716, Z=0.010)$ for the LMC and of $(X=0.726, Z=0.004)$ for the SMC. The average $M-L$ relation for the fundamental Cepheids matches closely that for the first overtone Cepheids for each Magellanic Cloud. Neither the SMC nor the LMC average $\log M-\log L$ relations are straight, but have a noticeable curvature. In view of the uncertainties in distance and reddening we have adopted three different choices for these quantities. The results based on the "long" distance scale to the clouds give a better agreement between theory and and observations than the "short" one. All the current evolutionary tracks predict systematically larger masses for given luminosities than our observationally derived ones, especially at the high end. Moreover, our study confirms that the evolutionary tracks of the low mass stars in SMC are not in agreement with the observations as they do not extend sufficiently blueward and do not penetrate deep enough into the instability strip, or not at all. The inference of masses directly from the observational database yields a novel and strong constraint on evolutionary calculations.
\end{abstract}

Key words. stars: oscillations - stars: Cepheids - stars: evolution, Magellanic Clouds, distance moduli

\section{Introduction}

In the last few years, high quality data on large numbers of Cepheid variables in the Small and Large Magellanic Clouds have been made available by the EROS and OGLE microlensing projects (Beaulieu et al. 1995; Afonso et al. 1999; Udalksi et al. 1999a-c). In particular the OGLE Project has provided standard colors in addition to periods and magnitudes for the largest samples published to date. In this paper we examine some of the constraints that the MC Cepheids impose on stellar evolution and stellar pulsation theories.

We use the full catalogue of publicly available of LMC and SMC single mode Cepheids and SMC double mode Cepheids produced by OGLE in BVI (Udalski et al. 1999a-c, with the zero point corrections as suggested in the April 2000 OGLE web site, U99 hereafter). The single mode Cepheid catalogues contain 1435 LMC and 2167 SMC stars. We keep the objects classified as fundamental mode pulsators or first overtone pulsators, with reliable photometry in both $V$ and $I$. We exclude stars whose magnitudes are most likely to be strongly contaminated

Send offprint requests to: J. P. Beaulieu, e-mail: beaulieu@iap.fr by companions or blending in $V$ or $I$. The remaining stars form our working sample of OGLE Cepheids. It consists of $670 \mathrm{LMC}$ fundamentals, $426 \mathrm{LMC}$ overtones, $1197 \mathrm{SMC}$ fundamentals and $677 \mathrm{SMC}$ overtones, as well as $24 \mathrm{~F} / \mathrm{O} 1$ SMC double-modes and 71 O1/O2 SMC double-modes.

The OGLE data base provides intensity averaged magnitudes and colors. With the help of distance moduli, these can then be transformed to luminosities and effective temperatures. The Magellanic Clouds (MC) are thought to be relatively uniform in composition, and with the observed average compositional information theoretical modeling can then provide the mass of each star.

We have to adopt distances and reddenings for the Magellanic Cepheids. The distance of the Large Magellanic Cloud remains at the center of the current debate about the distance scale ladder. Extreme values of the distance modulus range from 18.08 to 18.70 , but the distance estimates tend to cluster around 18.3 for the "short" distance scale, and around 18.5 for the "long" distance scale (see Walker 1999; Udalski 2000 and references therein; Cole 1998; Girardi 1998; Stanek et al. 2000; Romaniello et al. 2000; Feast \& Catchpole 1997; Luri et al. 1998; Groenewegen \& Oudmaijer 2000 and references therein; Groenewegen 2000; Sakai et al. 2000; 

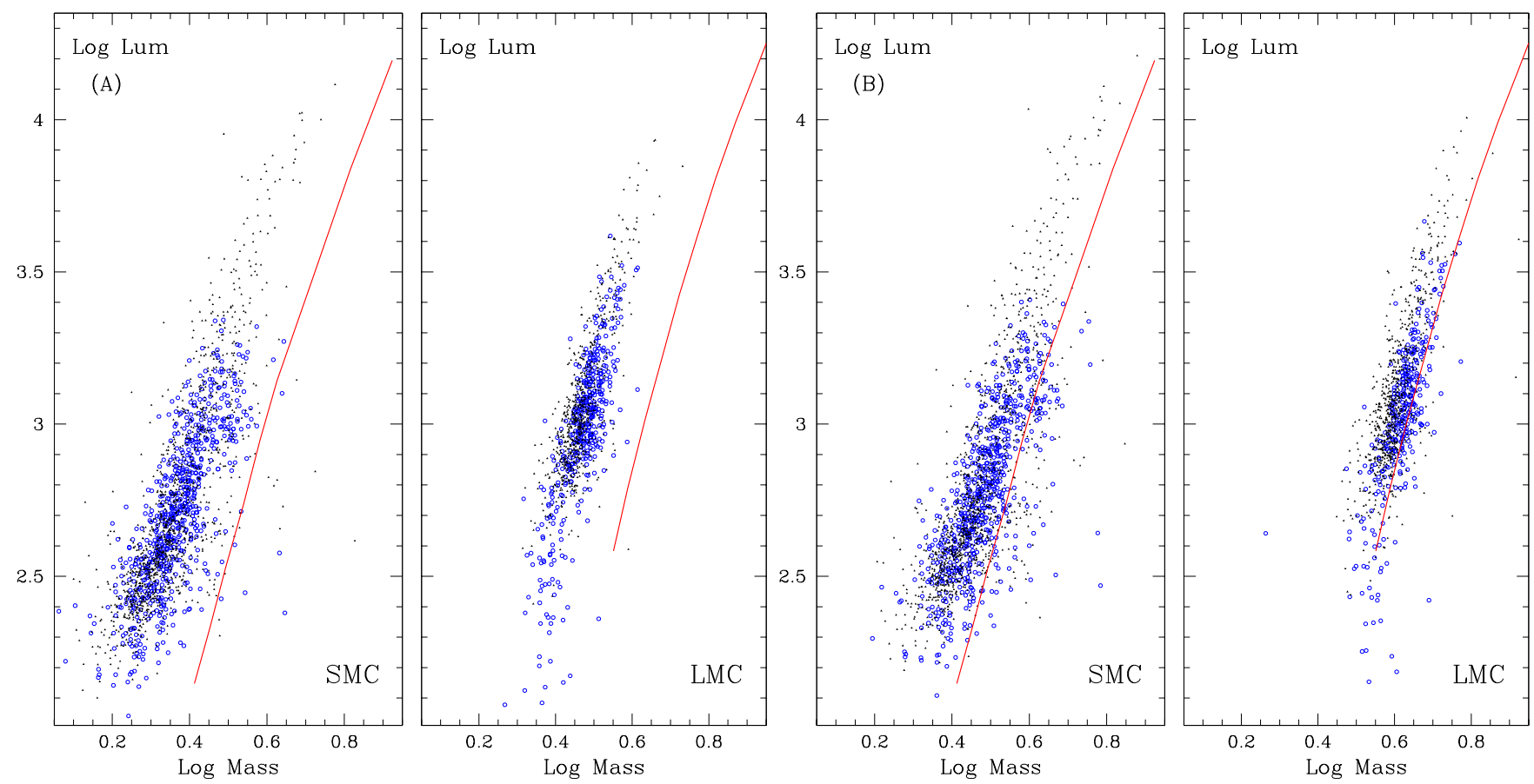

Fig. 1. Mass-luminosity relations for SMC and LMC as derived from the OGLE data adopting choice a) for distance modulus and reddenings in the left panel and our preferred choice b) in the right panel; fundamental Cepheids are shown as dots and overtones as open circles. As a guide to the eye we have superposed the evolutionary $M-L$ relations of Girardi et al.

Cioni et al. 2000). It is beyond the scope of the paper to solve this distance scale problem, but we will justify the choices we adopt for distances and reddenings.

U99 derive reddening by making the assumption that the red clump $I$ luminosity has a weak metallicity dependence, and they use the $I$ luminosity to map relative variations of $E(B-V)$. Finally they fix the zero point of the relative scales on the basis of observations of NGC 1850, NGC 1835, HV 2274 in the LMC, and NGC 416 and NGC 330 in the SMC. These assumptions lead to the determination of a "short" distance to the LMC of $18.24 \pm 0.05$ and $18.75 \pm 0.05$ to the SMC. The relative distance between LMC and SMC obtained with four different distance indicators (Cepheids, RR Lyrae, red clump and tip of the red giant branch) but with the same reddening maps, gives a relative distance modulus of $0.50 \pm 0.02$.

The reddening maps of U99 give a mean reddening for the LMC of 0.147 , and of 0.092 for the SMC. The consistency of the zero point of the reddening scale between these different observations is at the level of few times $10^{-2}$ (e.g., $E(B-V)=0.15 \pm 0.05$ for NGC 1850 and $E(B-V)=0.13 \pm 0.03$ for NGC 1835). We note that these values are different from what is usually given as mean properties for the clouds (especially for the LMC, see Walker 1999 and references therein). In his recent review, Walker (1999) noted that the median reddenings are $E(B-V) \sim 0.1$ for the LMC and $E(B-V) \sim 0.08$ for the SMC. The galactic foreground reddenings along the line of sight of the clouds are known to be low, viz. 0.06 and 0.04 , respectively. The estimation of differential reddening inside the clouds based on earlier studies is quite uncertain. In particular, heavily reddened stars $(E(B-V)=0.30)$ can be found all over the LMC, but the typical range is $0-0.15$.

In view of these uncertainties and controversies, we consider three alternative choices for the distance moduli and for the reddening corrections for our derivation of stellar parameters from the OGLE data:

Choice (A) adopts both the distance moduli and the reddening as suggested by U99.

Choice (B) adopts the Cepheid distance modulus to the $\mathrm{LMC}$ of $18.55 \pm 0.10$, and a relative distance between LMC and SMC of $0.42 \pm 0.05$ (Laney \& Stobie 1994), with the mean reddenings of $E(B-V)=0.1$ and $E(B-V)=0.08$ for LMC and SMC respectively.

Choice (C) is intermediate in that it adopts U99's relative distance between LMC and SMC of $0.50 \pm 0.02$ and its reddening, but a "long" LMC distance modulus of 18.55.

To summarize the differences, $(\mathrm{B})-(\mathrm{A})$, in distance moduli and in mean reddenings are $\delta \mu=0.31, \delta\langle E(B-$ $V)\rangle \sim 0.047$ for the LMC, and $\delta \mu=0{ }^{\mathrm{m}} 22, \delta\langle E(B-V)\rangle \sim$ 0.012 for the SMC. The differences, $(\mathrm{C})-(\mathrm{A})$, in distance moduli and in mean reddenings are $\delta \mu=0.25, \delta\langle E(B-$ $V)\rangle=0$ for the LMC and the SMC.

We follow Kovács (2000) in the conversion from magnitudes to bolometric, and from colors to effective temperatures. Because of the wider range of masses that are needed for this study, we have redone the Kovács fits with the Kurucz (1995) stellar atmospheres from the 


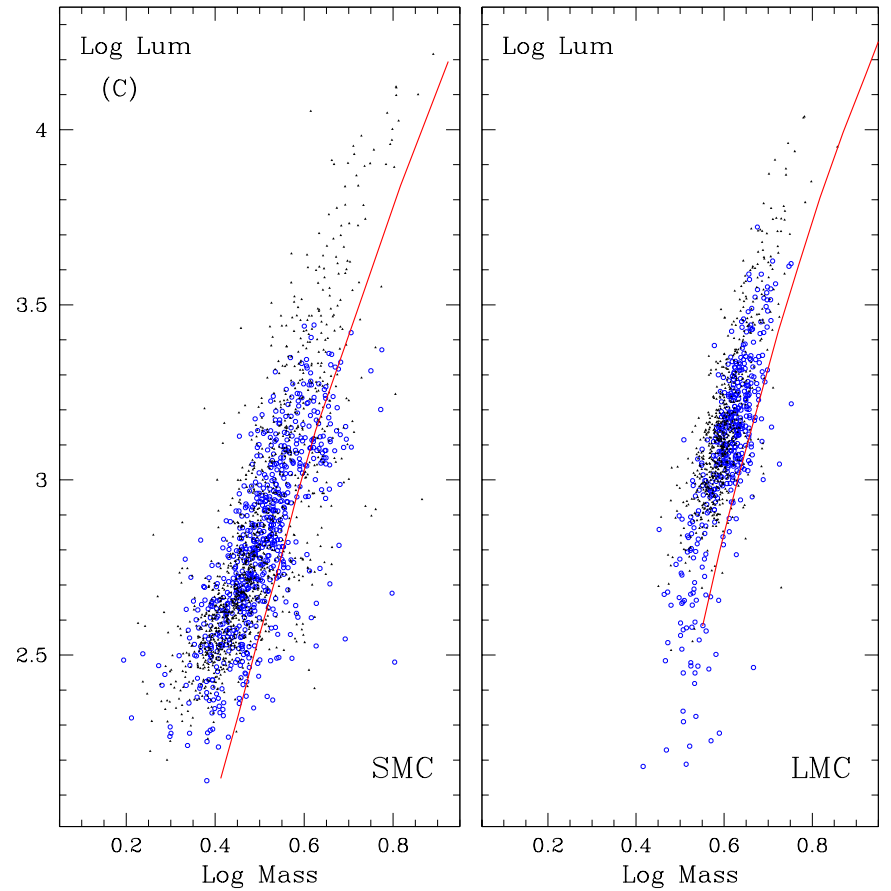

Fig. 2. Mass-luminosity relations for SMC and LMC as derived from the OGLE data with choice c) for distance modulus and reddenings. Superposed are the $M-L$ relations of Girardi et al.

BaSeL database (Lejeune et al. 1997) for the LMC and SMC chemical compositions for the temperature, luminosity and $\log g$ range appropriate for Cepheids ranging from $100 L_{\odot}$ to $20000 L_{\odot}$, with the results

$$
\begin{aligned}
2.5 \log L= & \mu_{\mathrm{MC}}-V+R_{V} E(B-V)+B C+4.75 \\
\log g= & 2.62-1.21 \log P_{0} \\
\mathrm{SMC}: & \\
\log T_{\text {eff }}= & 3.91611+0.0055 \log g \\
& -0.2482\left(V-I_{\mathrm{c}}-\left(R_{V}-R_{I}\right) E(B-V)\right) \\
B C= & -0.0324+2.01 \Delta T-0.0217 \log g \\
& -10.31 \Delta T^{2}
\end{aligned}
$$

LMC :

$$
\begin{aligned}
\log T_{\text {eff }}= & 3.91545+0.0056 \log g \\
& -0.2487\left(V-I_{\mathrm{c}}-\left(R_{V}-R_{I}\right) E(B-V)\right) \\
B C=- & 0.0153+2.122 \Delta T-0.0200 \log g \\
& -11.65 \Delta T^{2}
\end{aligned}
$$

where $\Delta T=\log T_{\text {eff }}-3.772$ and $L$ is in solar units. The transformation to absolute luminosities is then made with the adopted distance moduli $\mu_{\mathrm{MC}}$ to the LMC or the SMC for the various choices (A), (B) and (C). We note that compared to Kovács there are systematic shifts of $50 \mathrm{~K}$ in $T_{\text {eff }}$, and 0.02 in $\log L$.

It may be objected that since $\log g=\log (G M) / R^{2}$ in Eq. (2) there is an implicit assumption about a massradius -period relation. However, in Eqs. (3-6), $\log g$ appears with a tiny multiplier, and over the period range from 1 to 10 days its contributions to $\log L$ and $\log T_{\text {eff }}$ vary by 0.013 and 0.0046 , respectively. Ultimately, there is essentially no feedback on our mass determination.

\section{Mass luminosity relation}

With the help of relations (6) and the OGLE data we therefore obtain a period, a luminosity and a $T_{\text {eff }}$ for each fundamental and for each overtone Cepheid. These quantities are sufficient to compute the masses with the help of a stellar model builder and linear pulsation code, since $P_{0}=P_{0}\left(L, M, T_{\text {eff }}, X, Z\right)$ and $P_{1}=P_{1}\left(L, M, T_{\text {eff }}, X, Z\right)$.

For the computation of the SMC and LMC Cepheid models we have adopted the respective compositions $X=$ $0.726, Z=0.004$ and $X=0.716, Z=0.01$. We have used OPAL opacities (Iglesias \& Rogers 1996) merged with the low temperature ones of Alexander \& Ferguson (1994). Turbulent convection has been treated as described in Yecko et al. (1998). The convective parameters were chosen as in Kolláth et al. (2001), although, the precise values of these parameters will have very little effect on the periods.

In Fig. 1 we present the $M-L$ diagrams obtained from our Cepheid model calculations that use the observational constraints, A in the left panel, and B in the right panel. Fig. 2 displays the results for choice (C). The fundamental Cepheids are represented as dots and the overtone Cepheids as open circles. To guide the eye and for later reference we have also shown the $M-L$ relations of Girardi et al. (2000) for the Cepheids on the second crossing of the instability strip.

Four features stand out immediately. First, the three choices give very similar $M-L$ distributions, especially in slope and scatter, but choice (A) has a zero point that is in substantial disagreement with the evolutionary calculations. Second, the observations indicate a curved massluminosity relation. Third, the average $M-L$ for the fundamental Cepheids agrees with that of the overtones. Fourth, there is a huge scatter whose nature needs to be addressed, because the Cepheids form a homogeneous group, and one would expect all of them to fall on a very tight $M-L$ line.

\section{Discussion}

\subsection{Which choice for distances and reddening?}

We recall that the main difference between $(\mathrm{B})$ and $(\mathrm{C})$ is the adoption of mean SMC and LMC reddenings in (B) and (OGLE) reddening maps in (C). As we have seen in Figs. $1-3$ there is no significant improvement with the use of reddening maps, neither in the $P-L$ residuals, nor in the scatter in the final $M-L$ plots. Choices (B) and (C) are essentially equivalent except for a tiny systematic shift parallel to the distribution.

As seen in Fig. 1, with the observational constraints (A) there is a systematic shift to higher luminosities and higher temperatures for the observed stars. Taken at face value, choice (A) would indicate that evolutionary calculations are far from the representative which we deem 


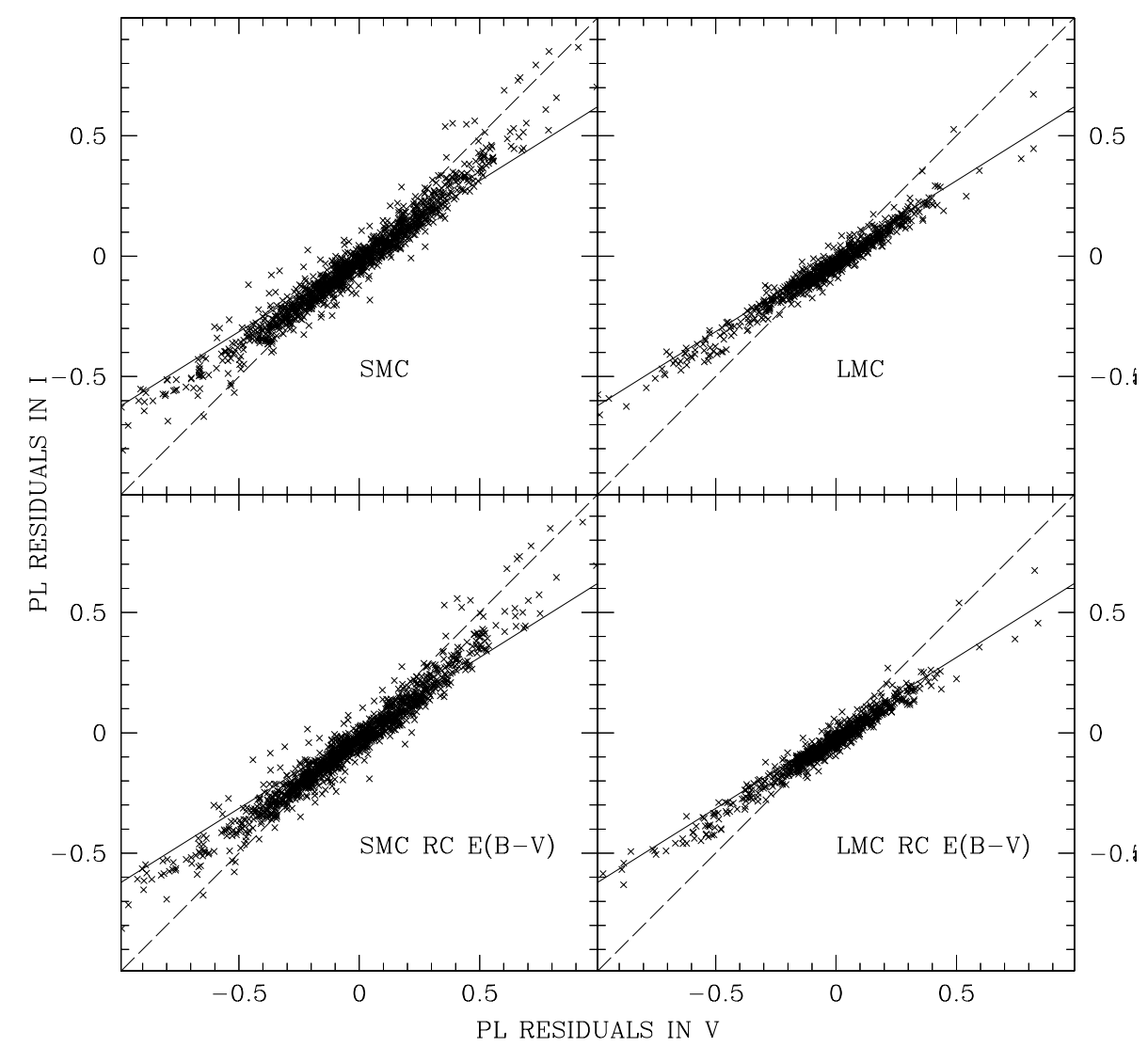

Fig. 3. The two upper panels gives the residuals of the $V$ and $I P-L$ relation for uncorrected reddening in LMC and SMC. The two lower are giving the residuals of the $V$ and $I P-L$ relation after reddening corrections based on the red clump stars following the U99 procedure. On each panel are indicated in solid the reddening line, and in dash the depth dispersion (the diagonal) line.

unlikely given the general agreement of several independent calculations (cf. Sect. 4). The sensitivity of the $M-L$ relations to metallicity would have to have been largely underestimated, too. On the other hand, with the "long" distances to the clouds of choices (B) and (C), the situation is much more satisfactory, as we shall see.

In the following, unless otherwise specified, we will adopt choice (B).

\subsection{Computational uncertainties}

First we examine the computational uncertainties. We expect these to be small because we compute only the linear periods of the fundamental and first overtone. In contrast to the linear growth-rates, the periods are very insensitive to the convective parameters ( $\alpha$ 's in Yecko et al. 1998). The comparison of purely radiative models with our turbulent convective ones gives an idea of the uncertainty. We find that the period shifts are systematic but small, of the order of the size of the dots in Figs. 1 and 2. The fact that they are systematic indicates that they cannot contribute to the scatter of Figs. 1 and 2. The models have been computed with a mesh of 200 points. Models run with a cruder mesh distribution give essentially the same $M-L$ picture. We can safely use linear periods, because nonlinear hydrodynamic modeling shows that the differences are systematic and at most of the order of $0.1 \%$ which has no appreciable effect on the $M-L$ picture.

We have not been able to find a computational uncertainty that can account for the scatter in the $M-L$ relation, and we have to look in the observational data.

\subsection{Scatter in the $M-L$ relations}

In the upper panel of Fig. 3 we plot the residuals of the period-luminosity $(P-L)$ relation in $V$ and $I$ for both the LMC and the SMC fundamental Cepheids OGLE data. These diagrams illustrate the structure of the Cepheid $P$ $L$ relation (see Figs. 5 and 6 from Sasselov et al. 1997). The dispersion is mainly along the reddening vector in the LMC, whereas in the SMC it is not because depth effects are another source of scatter. We recall (e.g., Sasselov et al. 1997) that there is an unfortunate near degeneracy between lines of constant period and reddening. Therefore one cannot just minimize the dispersion along the reddening vector in this plane to correct for the reddening. It would lead to an overcorrection. When we use the reddening derived by U99 we note a very marginal improvement of the residuals as shown in the lower panel of Fig. 3 and as noted by U99. 
We conclude that the differential reddening within the clouds on a star by star basis persists as a major source of dispersion that is not compensated for by the reddening maps from red clump stars.

In order to see whether the size of error that is inherent in the observations is responsible for the scatter in our $M-L$ relation we have made the following test. First we construct a sequence of fundamental Cepheid models with a specific $M-L$ relation, $\log L=0.79+3.56 \log M$, and with a range of $T_{\text {eff }}$ that spans the corresponding instability strip. With Eqs. (1-6) we transform $L$ and $T_{\text {eff }}$ to $I$ and $V$ magnitudes, and maculate these data with Gaussian noise of intensity 0.02 in the $I$ and $V$ magnitudes, and with a Gaussian noise in the reddening with $\sigma_{E(B-V)}=0.06$. Using these surrogate stars as input we then proceed to compute the surrogate stellar masses the same way as we handled the OGLE data. Figure 4 shows the resulting $M-L$ relation. It is seen to exhibit the same type of scatter as the OGLE derived $M-L$ relations.

This sensitivity can also be seen analytically. For that purpose we have made a rough fit with the help of our models

$\log P_{0}=11.80-0.595 \log M+0.82 \log L-3.55 \log T_{\text {eff }}(7)$

Together with Eq. (6) one then derives

$$
\begin{aligned}
\delta \log M & =0.51 \delta \mu-0.51 \delta V+1.37 \delta(V-I) \\
\delta \log L & =0.4 \delta \mu-0.4 \delta V+1.32 \delta E(B-V) .
\end{aligned}
$$

As a check, with the noise level 0.02 in the $I$ and $V$ magnitudes and 0.06 in the reddening for the preceding test one obtains $\delta \log M=0.05$ and $\delta \log L=0.09$, in agreement with the numerical results.

The reason for the scatter in the derived $M-L$ relation is thus seen to originate in the extreme sensitivity of the mass-luminosity relation to small photometric errors in the $V$ and I magnitudes.

It is very tempting to use the observational deviations in Fig. 3 to tighten the derived $M-L$ data. The question is whether we can use the deviations parallel to the reddening line to estimate (and correct for) the reddening and observational noise. We find that, because of the finite width of the instability strip, the spread in $T_{\text {eff }}$ has an effect parallel to the reddening, so we cannot decouple the reddening error from it. The spread in mass (for a given $L$ ) has an effect not parallel (at an angle close to 45 degrees) to the reddening, thus with a component perpendicular to the reddening line. Because of this projection angle the perpendicular direction alone cannot be used to estimate the observational errors in $I$ or $V$. In summary, it is unfortunately not possible to use the residuals of Fig. 3 to correct for the observational reddening errors on a star by star basis.

\section{Beat Cepheids}

OGLE have also published data on SMC beat Cepheids. Because the knowledge of a (precise) second period adds a

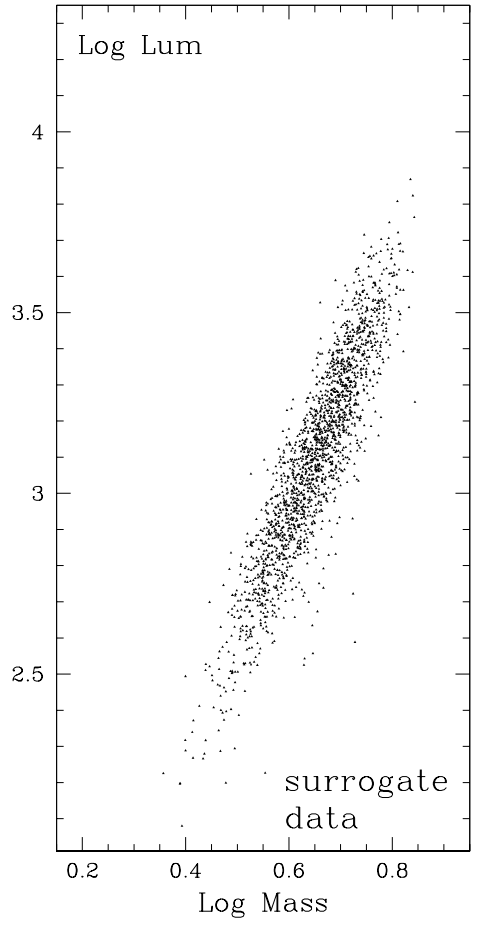

Fig. 4. Mass-luminosity relations for surrogate stars.

vital piece of information, these stars should be even more constraining than the single-mode Cepheids for extracting an $M-L$ relation. In fact Kovács (2000) has used the two observed periods and $T_{\text {eff }}$ and radiative linear Cepheid models to infer luminosities and thus the distance modulus to the SMC.

In order to check the self consistency of the observational data and pulsation models we can make the following test on the SMC beat Cepheids. We take three of the four observed quantities, viz. $T_{\text {eff }}, L, P_{k}$ and $P_{k+1}(k=0$ for the $\mathrm{F} / \mathrm{O} 1$ and $k=1$ for the $\mathrm{O} 1 / \mathrm{O} 2$ beat Cepheids). From these three parameters (ignoring $P_{k+1}$ for the time being) we calculate the mass and then the second period $P_{k+1}$ (calc). Then we compare this calculated period to the observed one $\left(P_{k+1}(\mathrm{obs})\right)$ in Figs. 6 and 7 . On the $\epsilon=P_{k+1}($ calc $) / P_{k+1}(\mathrm{obs}) \quad$ vs. $P_{k}$ diagram, with the choices $(\mathrm{A}, \mathrm{B}, \mathrm{C})$ of distance modulus and $E(B-V)$ we observe the following facts:

- For the F/O1 stars, the data fall along an almost horizontal line with little scatter. A similar result is obtained for the different choices, but $\epsilon$ is closer to 1 for choice (A). To get a self-consistent solution for these stars for choice (B), we have to increase the luminosity by $\delta \log (L)=0.12$. Decreasing the metal content $(Z)$ to 0.001 also shifts the $\epsilon$ values in the right direction, but by itself it does not solve the discrepancy;

- For the O1/O2 beat Cepheids, the $\epsilon$ values scatter around a line with a slope of $\approx 0.03$. There is only a limited range around $P_{1} \approx 1.0$ days, where self consistent solutions exist for the stellar parameters;

- The scatter on the $\epsilon$ vs. $P_{k}$ plots are consistent with the observational noise in $E(B-V), I$ and $V$. We note 

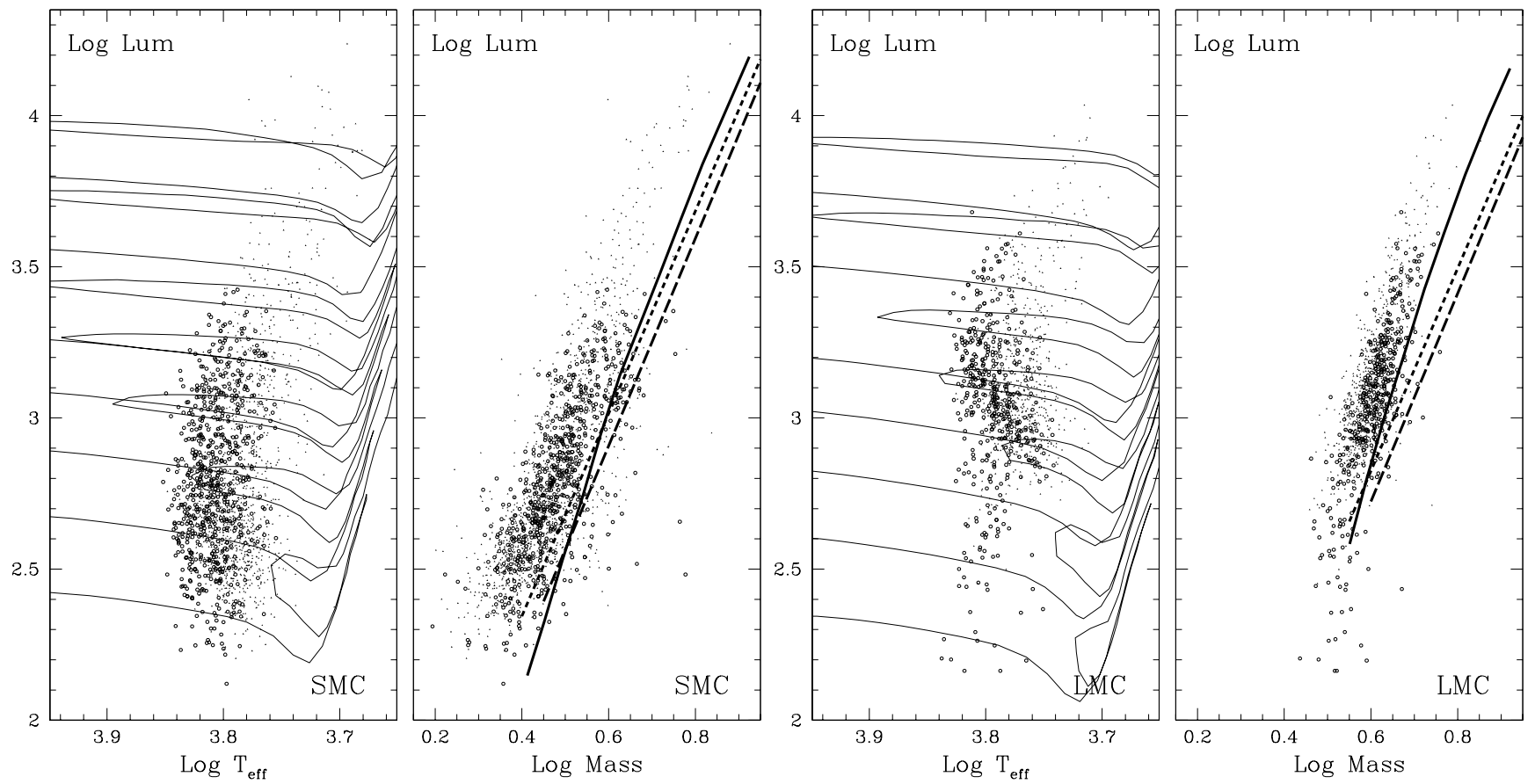

Fig. 5. Theoretical HR diagram and mass-luminosity relations for SMC (the two left panels) and for LMC (the two right panels). As in Fig. 1, fundamental Cepheids are shown as solid and overtones as open circles calculations: Theoretical HR diagram with superposed evolutionary tracks from Girardi et al. (2000). $M-L$ relations from evolutionary calculations; solid lines: Girardi et al. (2000), dotted lines: Alibert et al. (1999), dashed lines: Bono et al. (?).
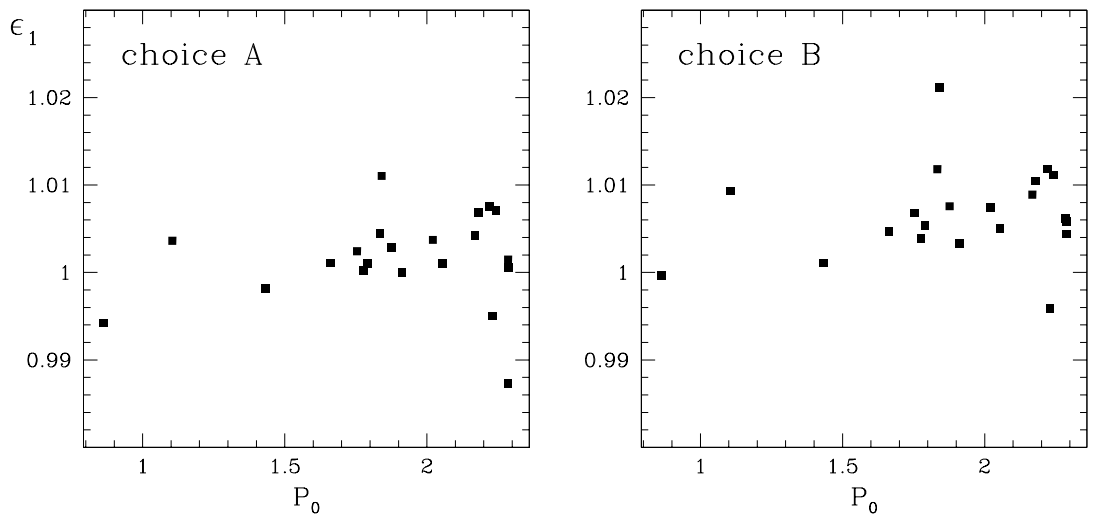

Fig. 6. SMC F/O1 stars: $\epsilon_{1}=P_{1}$ (calc) $/ P_{1}$ (obs) vs. the fundamental period for the SMC F/O1 stars. Left: distance modulus from choice $\mathbf{a})$, Right: distance modulus from choice $\mathbf{b})$.

that with the help of surrogate data with the same noise as described in Sect. 2.3, we found that these error sources do not introduce systematic trends (like the slope of $\epsilon$ ).

For the second set of tests we allowed systematic shifts in $\log L$. For the O1/O2 Cepheids, the slope of $\epsilon$ strongly depends on the adopted $\delta \log L$. With $\delta \log L=-0.05$ to -0.10 , the slope is removed but the scatter of the points is increased, and $\epsilon<1$ for all of the stars. Consistent solutions exist again only if the metallicity $(Z)$ is decreased to 0.001 . In the case of F/O1 stars the distance modulus has a less significant effect on the slope of $\epsilon$. The best agreement was found with $\delta \log L=0.10$ which is opposite to the value we found for the $\mathrm{O} 1 / \mathrm{O} 2$ Cepheids.

We have checked whether this discrepancy can be removed by allowing a wider range of initial assumptions on the input parameters. For our first set of tests the distance modulus was fixed, and we allowed a wide range in reddening $(-0.1<\Delta E(B-V)<0.1)$ as well as various changes in the composition and metallicity mixtures with the customized OPAL library. All these changes in the input data result in some vertical shifts in the $\epsilon$ vs. $P_{k}$ diagram, but not enough to get consistent solutions for the F/O1 stars. The metallicity would need to be decreased to $Z=0.001$ to get the mean value of $\epsilon$ to be 1 . We also note that there 

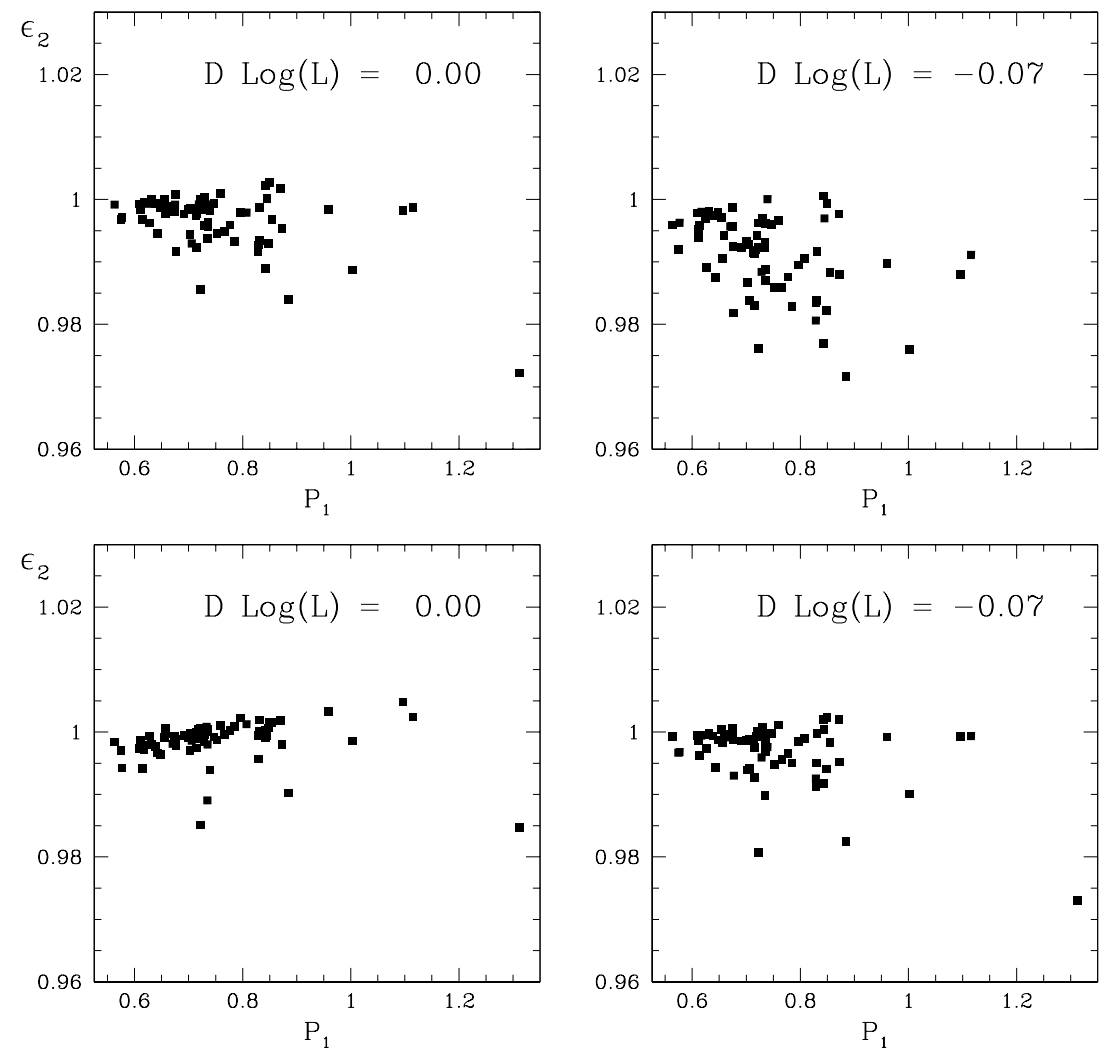

Fig. 7. SMC O1/O2 stars: $\epsilon_{2}=P_{2}$ (calc) $/ P_{2}$ (obs) vs. the first overtone period for the SMC O1/O2 stars. The upper pannels are for the choice a) of distance and reddening, whereas the lower pannels are for the choice b). Left: distance modulus from choice a) or b), Right: with $\delta \log L=-0.07$.

is no significant difference in $\epsilon$ between the radiative and convective models.

Our conclusion agrees with the work of Buchler et al. (1996), but is in apparent disagreement with Kovács (2000). The reason for this apparent disagreement is that Kovács did not construct models with the observational parameters, but simply minimized what he called $\sigma$, viz. his measure of the deviation from observed to model periods, and in fact this sigma is not zero for many of his "solutions". Furthermore in those cases where a solution can be found, the mass is determined with a very large uncertainty by the two period constraint, as already pointed out by Buchler et al. (1996).

Moreover, although this does not directly affect the absence of solutions, we remark that Kovács adopted reddening following that of U99. These reddenings are $\sim 0.01$ larger than the mean reddening towards the SMC. This will marginally affect his temperature scale compared to ours. However the distance he derives is not in agreement with the distance adopted by U99 to the SMC, but is close to ours.

We note that the same trouble arises when we use the 3 observational data, $\left(P_{k}, P_{k+1}, T_{\text {eff }}\right)$ and compute $L$ and $M$. For many stars in the SMC sample there is no solution, i.e. no mass and luminosity can be found that satisfies these three observational constraints! The same difficulty appears when, instead, one tries to satisfy the 3 observational constraints $\left(P_{k}, P_{k+1}, L\right)$ to compute a $T_{\text {eff }}$ and $M$

In the cases where there are solutions based on three pieces of observational data, they are generally not compatible with the fourth one, i.e. if the periods and $T_{\text {eff }}$ are given, the calculated luminosity and mass are not fully acceptable. Why there are no satisfactory solutions for the observed beat Cepheids in the SMC remains an unsolved puzzle that the introduction of turbulent convection in the linear codes has not resolved.

\section{Comparison to evolutionary tracks}

It is of course of great interest to confront the predictions of evolution calculations with the $M-L$ values which we have extracted from the OGLE data. Recently a number of such calculations, all performed with the OPAL opacities, have become available: Alibert et al. (1999), Girardi et al. (2000), Bono et al. (?). Alibert et al. have also compared their results to observational EROS $P$-magnitude-color values and have obtained reasonable agreement. Of course our results, by construction, satisfy these observational constraints.

The evolution calculations have not been compared to the SMC and LMC OGLE-derived stellar parameters, neither in a $\mathrm{HR}$ diagram nor in a $M-L$ diagram, both of which we now present in Fig. 5. We plot the evolutionary tracks of Girardi with $(X=0.756, Z=0.004)$ and 
( $X=0.742, Z=0.008)$, respectively, which are the closest to our chosen compositions. The Girardi et al. $M-L$ relations for the $2 \mathrm{nd} / 3 \mathrm{rd}$ crossing (taken as the points of slowest evolution near the blue edge) are shown as solid lines, those of Alibert et al. as long dashes and those of Bono et al. with $(Y=0.226, Z=0.004)$ and $(Y=0.216$, $Z=0.004)$ as short dashes.

We note that none of the three sets of evolutionary calculations is fully in agreement with our OGLE-derived LMC and SMC $M-L$ data (nor are the older calculations), even when we adopt the most favorable choice of distance modulus and reddening. At fixed mass, the evolved stellar models are not luminous enough. The results of Girardi et al. are closest to our derived $M-L$ relations, and they also seem to have the right curvature (Alibert et al. and Bono et al. used straight line $M-L$ fits). Indeed, if the $M-L$ of Girardi et al. are shifted by $\sim 0.35$ in $\log L$ for SMC (respectively by $\sim 0.25$ in $\log L$ for LMC) metallicities, a reasonable agreement obtains at low and high luminosities. This could be achieved, at least partially, with overshooting or an increase thereof (Baraffe, priv. comm.).

We have not shown the $M-L$ relations for the faster, first crossing to avoid cluttering the figures. It can be seen from the left-hand sub-figures that the luminosities are about 0.2 lower for the same mass on these crossings.

The density of stars is definitely lower at the low luminosity end. A natural explanation is that the low luminosity stars are first crossers. The Girardi et al. tracks for the LMC are compatible with this interpretation, but it would be useful to do the statistics on the basis of the evolution speed along the tracks. However, for the SMC, even the Girardi $M-L$ is much too low for the first crossers.

Finally, the Girardi low $L$ tracks do not loop sufficiently far for the SMC. The problem is slightly worse for both the LMC and SMC tracks of Alibert et al..

The reader may wonder why both the evolutionary calculations and our pulsational calculations claim to give agreement with the observational data, yet they are based on substantially different stellar masses. To discuss the origin of this discrepancy we first need to compare the two procedures.

In our calculations, we rely only on observed periods, colors and magnitudes, transformed to $T_{\text {eff }}, L$ with Kurucz models, and from which we construct stellar models that exactly satisfy these observational constraints. The computed periods are essentially independent of any physical and numerical uncertainties, as pointed out in Sect. 3.2. Furthermore, we do not rely on the stability of the models. The latter are quite sensitive to physical uncertainties, especially the turbulent convective parameters. (We note though that, with our "standard" parameters, the vast majority of our models are linearly unstable.)

In order to determine the $T_{\text {eff }}$ range of Cepheid behavior along the evolutionary tracks, Alibert et al., for example, performed a linear stability analysis along these tracks and then compared the properties of their unstable models to the observational EROS $P$-magnitude and $P$-color data sets. The use of a stability analysis with the inherent uncertainties coming mostly from turbulent convection necessarily introduces an uncertainty in the temperature range of the Cepheid models (width of the instability strip).

Equation (7) gives a clue to the apparent discrepancy: It is possible to absorb rather large changes in $M$, say $\delta \log M=0.1$ (the largest difference between the evolutionary calculations, or with our average $M-L$ ), with a tiny change in $\log T_{\text {eff }}$ of $\delta \log T_{\text {eff }}=0.02$. This shows that our procedure of extracting masses directly from the observational data can therefore impose a novel, external and stronger constraint on the evolutionary calculations than is available from the previous comparison to observed $P$-magnitudes and colors.

\section{Conclusions}

We have used the OGLE data base and Kurucz atmosphere models to obtain periods, effective temperatures and luminosities for fundamental and overtone Magellanic Cloud Cepheids. With an assumed average composition of $(X=0.716, Z=0.010)$ for the LMC and of $(X=0.726$, $Z=0.004)$ for the SMC, stellar masses have been computed with the Florida pulsation code for the observationally derived $P, L$ and $T_{\text {eff. }}$ The best results are obtained by adopting the "long" distances to the clouds. Reddening maps, as opposed to average MC reddenings, do not reduce the unexpectedly large scatter in $M-L$, and we conclude that individual stellar reddenings would be necessary. However, the largest source of the scatter is in the $V$ and $I$ photometric errors. The $M-L$ relations for the fundamental and for the first overtone Cepheids match closely for each Magellanic Cloud. Both the SMC and the LMC $\log M-\log L$ relations have a noticeable curvature.

A comparison with the predictions of the recent stellar evolution calculations show a discrepancy in the $M-L$ diagrams, where the evolutionary tracks are underluminous. It also confirms a discrepancy in the theoretical HR diagrams, especially for the SMC, where the low mass tracks do not extend sufficiently blue-ward to penetrate the instability strip. Our $M-L$ relations are useful as a new type of constraint that the evolutionary tracks have to satisfy.

Acknowledgements. This work has been supported by the National Science Foundation (AST98-19608) and by the Hungarian OTKA (T-026031). It is based on observations coming from the public archive from the OGLE-II microlensing experiment. We have profited from a fruitful correspondence with Géza Kovács, Isabelle Baraffe, Marie-Jo Goupil, Daniel Cordier, Giuseppe Bono. We thank Andrejz Udalski for his comments on the paper. Two of us (JRB and ZK) gratefully acknowledge the hospitality of the Institut d'Astrophysique de Paris, as a "Professeur invité de l'Université Paris VI" (JRB), and as a "Poste rouge" (ZK).

\section{References}

Alibert, Y., Baraffe, I., Hauschildt, P., \& Allard, F. 1999, A\&A, 344,551 
Afonso, C., et al. 1999, A\&A, submitted http:// www-dapnia.cea.fr/Phys/Spp/Experiences/EROS /Cepheides/catalog_cep.html

Afonso, C., et al. 2001, A\&A, submitted

Alexander, D. R., \& Ferguson, J. W. 1994, ApJ, 437, 879

Beaulieu, J. P., et al. 1995, A\&A, 303, 137

Bono, G., Caputo, F., Cassisi S., et al. 2000, ApJ, 543, 955

Buchler, J. R., Kolláth, Z., Beaulieu, J. P., \& Goupil, M. J. 1996, ApJL, 462, 83

Cioni, M. R., et al. 2000, A\&A, 359, 601

Cole, A. A. 1998, ApJL, 500, 137

Feast, M., \& Catchpole, R. M. 1997, MNRAS, 286, L1

Girardi, L., et al. 1998, MNRAS, 301, 149

Girardi, L., Bressan, A., Bertelli, G., \& Chiosi, C. 2000, A\&AS, 141,371

Groenewegen, M. A. T. 2000, A\&A, 363, 901

Groenewegen, M. A. T., \& Oudmaijer, R. D. 2000, A\&A, 356, 849

Iglesias, C. A., \& Rogers, F. J. 1996, ApJ, 464, 943
Kovács, G. 2000, A\&A, 360, L1

Kolláth, Z., Buchler, J. R., Szabó, R., \& Csubry, Z. 2001, A\&A, in preparation

Kurucz, R. L. 1995, Kurucz CD-ROM No. 23, Cambridge, Mass: Smithsonian Astrophysical Observatory

Laney, C. D., \& Stobie, R. S. 1994, MNRAS, 266, L441

Lejeune, T., Cuisinier, F., \& Buser, R. 1997, A\&AS, 125, 229

Luri, X., et al. 1998, A\&A, 335, 81

Romaniello, M., et al. 2000, ApJ, 530, 738

Sakai, S., et al. 2000, AJ, 119(3), 1197

Sasselov, D., et al. 1997, A\&A, 324, 471

Stanek, K. Z., et al. 2000, Acta Ast., 50, 191

Udalski, A., et al. 1999a, Acta Ast., 49, 223

Udalski, A., et al. 1999b, Acta Ast., 49, 437

Udalski, A., et al. 1999c, Acta Ast., 49, 1

Udalski, A. 2000, ApJL, 531, 25

Walker, A. 1999, in Post-Hipparcos Cosmic Candle, ed. A. Heck, \& F. Caputo (Kluwer Academic Publisher), 125

Yecko, P., Kolláth, Z., \& Buchler, J. R. 1998, A\&A, 336, 553 